\title{
Effect of investigationVPN to Secure Wireless LAN
}

\author{
El Mutaz Mahdi Osman Elawad, Dr.Haala El Dawo, \\ Department of Communications, Faculty of Engineering, Al-Née lain University
}

\begin{abstract}
Manycorporationsare seriously concerne dabout security of network sand the refore, the ir network superviso rsarestill reluctanttoinstallWLANs.In thisregards,theIEEE802.11istandard wasdevelopedto addressthesecurityproblems, eventhoughthe mistrustofthewirelessLANtechnologystill exists. The thought wasthatthebestsecuritysolutionscouldbefoundin openstandards based technologies that can be delivered by Virtual Private Networking(VPN)beingusedfor a long timewithoutaddressingany security holes for the pas tfew years.This work, addresses this issue and presentsa simulatedwirelessLANof IEEE802.11gprotocol,and analyzesimpactofintegratingVirtualPrivateNetworktechnologyto securetheflowof trafficbetweentheclientandtheserverwithinthe LAN, usingthe OPNET WLAN utility. Two Wireless LAN scenarios havebeenintroducedandsimulated.Thesearebasedon a normal extensiontoawired network andVPNover extension toawired network.Theresultsofthetwoscenariosarecomparedandindicate the impact ofimproving performance, measured byresponse time andload,ofVirtualPrivateNetworkoverwirelessLAN.
\end{abstract}

Keywords: IEEE802.11,VPN,Networking,SecureWireless, WLAN,Opnet.

\section{Introduction}

WIRELESS LAN technologies such as IEEE802.11provide endusers and network Gooddegree of flexibilityandcostreductionintermsofcost ofsavingcables[1-3]. However, withtheincreasedreliance OntheWLANs, the security issue is becoming of great concernforthistechnologyasit isbecominga subjectto numerousattacks.The se attacks oftendividedin to passive attac kssuchas eavesdroppingandtrafficanalysis,andactive attackssuc has Do Sand Masquerade.

Thissecurityweaknessof WLANs,leadsthe network vendorsandanalyststo lookforandprovideremediesto these attacksandthreats[4-5].Mostofthemagreethatthereare twoprimary levelsofsecuring forawireless network. The firstlevelis theFramelevelwhichintroducesencryptionand authenticationtechnologies, andthe secondlevelis theradio frequencylevel whichintroducesintrusiondetectionand prevention.

Thispaperisanattempttodefineenhancementofdifferent

H.Bourdoucen,A.AlNaamanyandA.AlKalbaniarewiththeElectrical and Computer Engineering Department, College of Engineering, Sultan QaboosUniversity,P.O.B;ox33, Al-Khod,Muscat123,Sultanateof Oman (authore-mails: hadj@squ.edu.om,naamany@squ.edu.om,m051407@ squ.edu.om).

Technologiesusedto secureandreducethethreatsassociated with the wireless LAN, and examines the impact of using VirtualPrivateNetworktechnologyto securetheWLAN technology.In additiontothese, it will presentanalysisofits impact on the costaswellasonperformancemeasurements that are mainly related to delays and Load. After a numberofobservedvulnerabilitiofonWEP,it was suggestedto go aheadwithdeployingWLANdiscounted securitymeasuresintroducedby theIEEE802.11standard working groups together with the Wi-Fi alliance. The idea wasthatthebestsecurity solution couldbefoundinopen, standardbasedtechnologiesdeliveredby VirtualPrivate Networking (VPN). IPsecurity (IPSec) isthestandard for VPN, which wentthrough anumber ofrevisions thathave resultedina robustsecuritystandardthatprovidesgooddata confidentiality, authentication, andaccess control regardless of the transmission medium. By integrating wireless LANs intoanIPSecinfrastructure, allowsWLANinfrastructure to focuson simplytransmittingwirelesstraffic, whilethe VPN wouldsecureit,asshowninFig.1below.

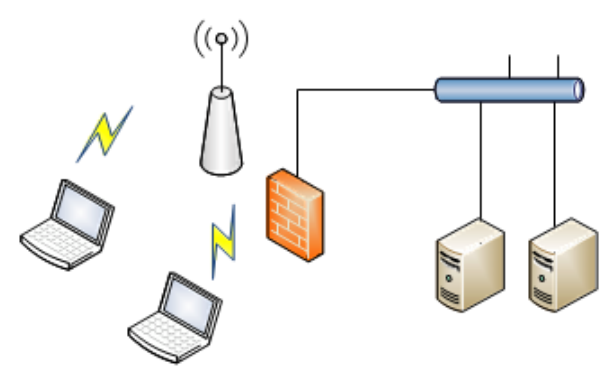

Fig.1 IntegrationofWLANandVPNconfiguration. 
NotethatthisVPNmaintainsdataprivacythroughtheuse tunnelingprotocolandsecurityprocedures.Thereare differentwaysonecanadopttoimplementa VPN,butthetwo mostcommon typesareremoteaccessVPNandsite-to-site VPN[6]. TheRemoteAccessVPNconfiguration isusedto allowVPNsoftwareclientssuchas mobileusersto securely accesscentralizednetworkresourcesthatresidebehinda VPN server.The site-to-siteVPNallowsto creatededicated,secure connections between locations across the open Internet or public connection. They can be either Intranet-based or Extranetbased.Initssimplestform,byencryptingdatawhile it is sent and decrypting it at the receiver, the data is effectivelysentthrougha "tunnel"thatcannotbe"entered"by data that is not properly encrypted and part of the Communications process.Itinvolvesplacingapacketwithin anotherpacket, andsendingitovera network. Theprotocolof theouterpacketis understoodbythenetworkat bothpoints, calledtunnelinterfaces, wherethe packetentersand exitsthe network[6].

Datatransmittedover unsecuredcommunicationusing wirelesstechnologyby broadcastingsignalswhichcanbe receivedby manyhackers, whereendusersabsolutelycannot controlunlessthedatais encryptedthenit is extremely vulnerable to be intercepted or interrupted [7]. Besides,security,flexibilityis anotheradvantageofVPN'sas mostof them unableto carry almost all IP protocols according to IPSecstandard.Suchas Webservers,Emailservers,FTP servers,fileserversorDNSserverscanall be completel

accessedfrom anywherethroughVPNnetworks.Thisallows rationalizingresourcesandinformationto preventwaste.The availabilityofIPSecVPNcanbeusedbyclientstoconnectto thecompanyVPN,evenit comesfroma dial-connection[7]. However,somedisadvantages ofVPNneedtobeindicated, despiteoftheirpopularity.Theyin fact require adeep understanding ofpublic network security issues and need to takeproperprecautionswhile deployment.Also, VPN technologies ofdifferentvendorsmaynotworkwelldueto

noncompatibilityistheirspecifications.Tunnelingis theprocessofencapsulatingprivateIP packets intoanIPSecpacket,ina waythattheprivatedatapacketis embeddedintheIPSecpacket,asshowninFig.2below.

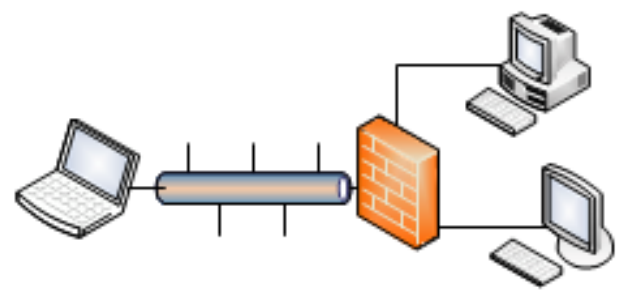

Fig.2 VPNVirtualtunneling

below,showstheimplementationofSSL[6].

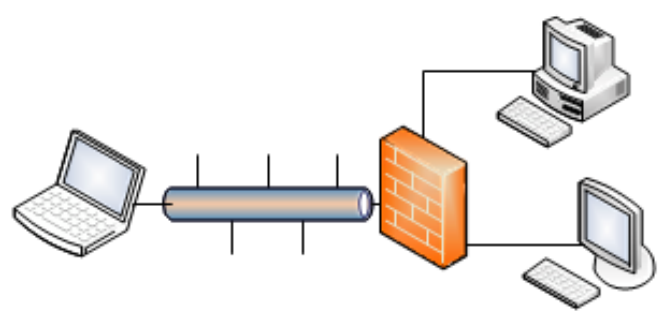

Fig.3 SSLtunnelinginaVPN

SinceSSLsupportisbuiltintowebbrowsersandmoste- mailclientprograms, thereisnoneedfora separateprogramto support these applications [8]. However, wireless LAN (WLAN)technologiessuchas 802.11gprovideend-usersand networkprofessionalsa tremendousamountofflexibility. However, While full information security may not be attainable, most experts agree that WPA and 802.11i representsan excellentsolution,exceptforone detail.Thisis relatedtothefactthat802.11dealsonlywiththe securityof the Airlink, whichis the portion of the information value chainbetweena givenclientanda givenaccesspoint.Thismeans, onlya secureconnectionbetweenwirelessclientandWLAN infrastructure[9].

Avirtualprivatenetworkcanprovidesecurityfarbeyond theairline. WhiletherearemanyformsofVPNs, a popular solution istouse the industry standard IPSecurity(IPSec) protocol, which is specified as part of the overall suite of InternetProtocols.IPSecallowsa mobileclientoranywired clienttoestablisha secure"tunnel"throughallthenetwork elementsbetweenclientandserver[10]. 


Belowisdescribeda frameworkforsecuringWLANtraffic usinga network-
layervirtualprivatenetwork(VPN).The network architecture foraVPN-secured WLAN implementationisshownbelowinFig.4.

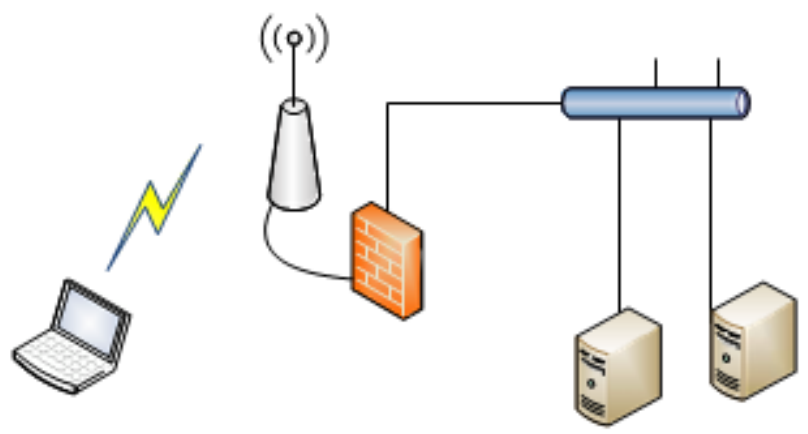

Fig.4 NetworkarchitectureforaVPN-securedWLAN

Theauthenticationbetween

$$
\text { the VPN gateways hasestablished }
$$

thetunnelandthe userscansendandreceivedataacrossit. IPSectunneltrafficatthepacketlevel,i.e.,atthenetwork layerof the OpenSystemsInterconnect(OSI)seven-layer model,andisindifferent towhichhigher-level protocol the packetsrepresent.BecauseanIPSecVPNencapsulatesall

packetsregardlessoftheirfunction,itautomaticallysupports all applicationsthatcommunicateusingIP.IPSecusually requirestheuseofaninstalledprogramontheclientmachine tohandletheencryption[8].

SecureSocketsLayer(SSL)VPNsencapsulatesdata traffic overanencryptedtunneltoa gateway,byinvokingSSL technology whencommunicating over an "HTTP secure" (https) web link. The receiving SSL gateway decrypts the trafficandpassesit totheinternalnetwork.SSLVPNstunnel trafficat thesessionlayeroftheOSImodel,butnotat the networklayer,sobydefaulttheyonlysupportsomespecific IP applications - typically web access and e-mail, Figure 3The primarycomponentofthiswirelessLANsecurity architectureis VPNConcentratorwhichistheVPNkey component. WhilethenontrustedoftheWLANsecurity, the useofVPNtechnologiessuchasPPTP,pureIPSec,or L2TP overIPSecprovidesbetterencryptionlevelsand dynamickey exchangesthatmitigatetheweaknessesofWEP.Inaddition, theauthenticationrequiredby theVPNaddsanotherlayerof controloveraccesstotheproductionLANbywirelessclients.

$$
\text { Dependinguponthe VPNConcentratoror firewallused, the }
$$

canbeintegratedintoservicesavailable ontheWiredLAN(RefertotheFig.

VPNauthentication 4).

Oncea userisauthenticated,alltrafficonthewireless network is encrypted and becomes safe from prying eyes, evenifa hackermanagedto penetratewirelessaccesspoint's securityandjointhenetwork, it wouldseenoclear-texttraffic because all legitimate users would be using an encrypted tunnel[11].

\section{Simulationresultsanddiscussion}

Two computer tools have been investigated

NS2 and OPNETforsimulationofWLANsecurityusingVPN.NS2is a discrete event simulator targeted at networking research, whichprovidessubstantialsupportforsimulationof TCP, routing, and multicastprotocolsoverwiredandwireless(local andsatellite)networks[12,13].But for this work,OpnetModeler11.0toolshave been used.Theparametersand configurationsneededforthe simulatedscenarioswillbe discussed.TheaimistoprogramandsimulatewirelessLAN of802.11 gprotocol,andanalyzetheimpactof integrating Virtual PrivateNetwork technology to secure the flow of traffic betweentheclientandtheserverfarm withinthe Local AreaNetwork, usingthe OPNET WLAN utility.

TheMarkovmodelforthe distributedcoordinationfunction (DCF)protocolhas beenshownto predictaccuratelythe networkthroughputof 802.11networksunderrealistictraffic load.Theperformanceof 802.11DCFhasbeenstudiedinthe literaturethroughvariousmodels, differentsimulations, and a numberof experiments [15-17].Thethroughputmodel developedby Bianchi providesan accurateandsimple analyticalmodelfor afinitenumberofterminalsandideal channelconditions[16].

TheprobabilitythatagivenstationtransmitsisexpressedAs:

Thispaper, thestatisticsofinterestaremainly:

EthernetDelay:whichrepresentsthe endto enddelayofall packetsreceivedbyallthestations.VPNDelay: whichgives theEnd-to-Enddelayfortrafficthrougha VPN.Thisdelayis measuredas the timeelapsedbetweentrafficenteringthenetwork through Ingressand traffic leaving the network through Egress. VPN Load: measures the amount of VPN-traffic enteredtheNetworkthroughIngress. Thestatisticismeasured in 
bitspersecond.VPNthroughput:measuresthe amountof VPN-trafficleavingtheNetworkthroughEgress. Thestatistic ismeasuredinbitspersecond.

WLANDelay:Representstheendto enddelayof all the packetsreceivedbythewirelessLANMACsof allWLAN nodesin thenetworkandforwardedto the higherlayer.This delayincludesmediumaccessdelayat thesourceMAC, receptionof all thefragmentsindividually,andtransfersof the framesviaAPwhileAccessPointFunctionalityenabled,

WLAN Throughput: bits (in bits/Sec) forwarded from wirelessLANlayerstohigherlayersinallWLANnodesof the network. WLAN Load: Represents the total load (in bits/Sec)submittedto wirelessLANlayersby all otherhigher layersinallWLANnodesofthenetwork.

DatabaseEntryforTrafficReceived:representsthe average bytespersecondforwardedto all

DatabaseEntryApplications bythetransportlayersinthenetwork.TrafficSend:whichis theaveragebytespersecondsubmittedtothetransportlayers byallDatabaseEntryApplicationsinthenetwork.

EmailTrafficReceivedTraffic:Averagebytesper

second forwardedtoallemailapplicationsbythetransportlayersin thenetwork. SendTraffic:Averagebytespersecondtraffic submittedtothetransportlayerswithallemailapplicationsin thenetwork.

Two scenarioswere simulatedfor seven hours duration,(about12hoursrealtime)arScenario(1):NormalWirelessLAN

$$
\begin{gathered}
\frac{1-p}{\tau=} \\
1-p \\
\times b_{0,0}
\end{gathered}
$$

(1)- Scenario (2): Wireless LAN with Virtual Private NetworkTechnology

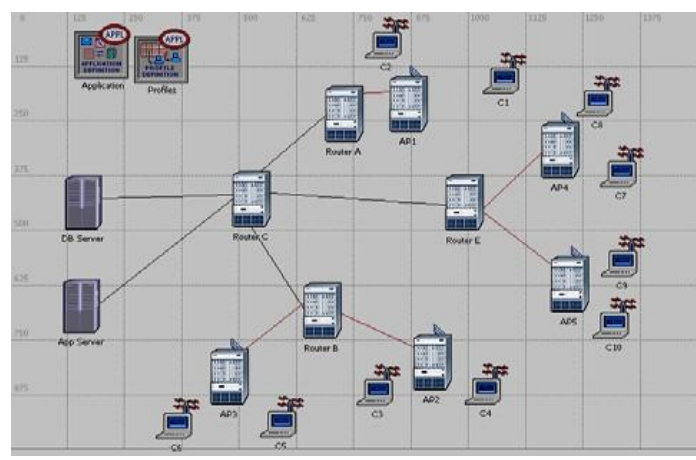

Where,pistheconditional collisionprobability andmis maximumbackoffstage. b0,0isexpressedas $b_{0,0}$

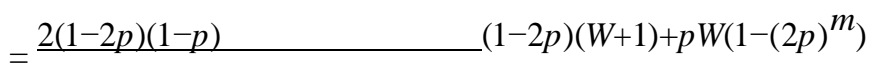

(2)WhereWisthecontentionwindow.

ThederivednormalizedsystemthroughputSisgivebythefollowingratio $S=\underline{P_{t r}} \underline{P}_{\underline{S}} \underline{E[P]}\left(1-P_{t r}\right) \sigma+P_{t r} P_{S} T_{S}+P_{t r}\left(1-P_{S}\right) T_{c}$

(3) Where $\mathrm{T}_{\mathrm{S}}$ istheaveragetime, $\mathrm{T}_{\mathrm{C}}$ thecollisiontime, and $\sigma$, slottimeandE[P]isthePayloadina slottime. Notethatthese fourparametersareconstant.Alsointhe equation(3)above, $\mathrm{P}_{\mathrm{S}}$ is theprobabilitythatatransmissionissuccessful, $\mathrm{P}_{\mathrm{tr}}$ theprobabilitythatthereis at leastonetransmissioninthe consideredslottime.

Therearemanystatisticsthatcanbe determinedafter simulating and designing VPN in OPNET environment. In

Fig.5(a)NormalWirelessLAN 


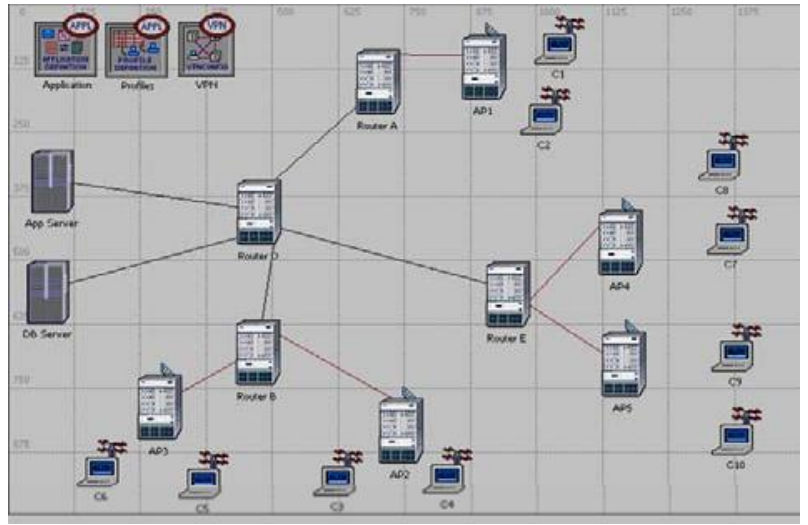

Fig.5(b)VPNoverWirelessLAN

Theobtainedresultsfromdifferentscenariosareanalyzed to measure the performance implication of deploying VPN over Wireless LAN, in which are divided into three categories:GlobalResults,LinkResults, andObjectResults.

Wirelesslocalareawithand withoutVPN have been simulated and results analyzed for both networks. The diagramsconsideredareshownFigure5(a),(b).

Partsofthesimulatedscenariosconsistof Ethernet connectivity (refertofigure5(a)), asaglobalresultofthe Ethernetdelayshowninfigure6below.

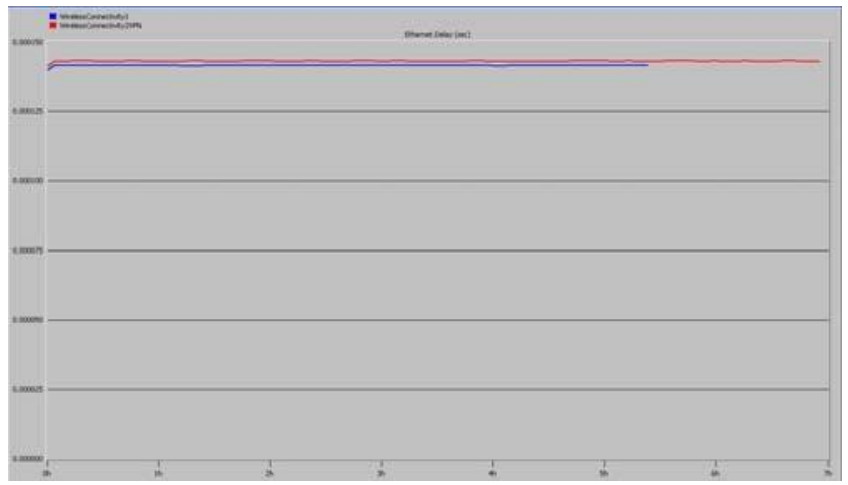

Fig.6EthernetDelay

Theresults indicatethatthereisnoimportant impact of a VirtualPrivateNetwork(VPN)overtheresponseof Ethernet delay.Theresultsshowrather stabledelayfor both normal andVPNscenarios, atapproximately0.14msec.

Theconsiderationsof datadroppedresultsindicatethe overflow traffic over thewireless object. The graphofthe results is showninFig.7. 
Fig.7 EthernetDelay

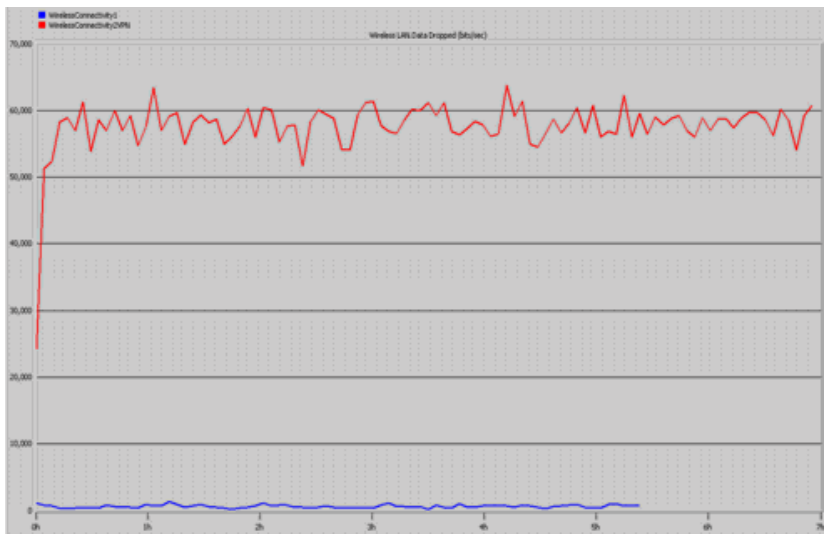

Unfortunately,the impactof theVPNoverwirelesstraffic indicates a high data drop, which is mainly due to the overflow of traffic. The observed data dropped differences show anaverage ofmorethan95\%.Forasimulation of4 hours run time, the data dropped at VPN is about 57.5

Kbits/Sec,andthenormalnetworkwithnoVPNshowsabout

$858 \mathrm{bits} / \mathrm{Sec}$.The resultsalsoindicatethat the datadrop continuesalmostatastableaveragerateof55Kbits/Secwhen aVPNisused.

Thewirelessdelaypresentsthedelayofpacketsreceived andforwardedby the wirelessnodesacrossthe network, the graphobtainedrepresentthe delaydifferencewithandwithout VPN,refertoFig.8.

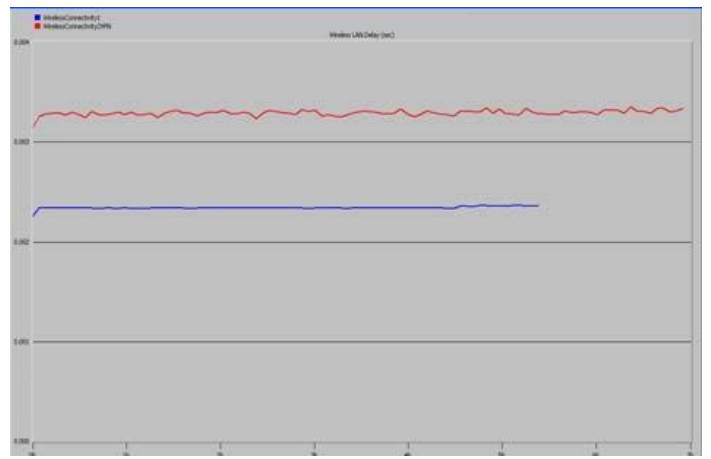

Fig.8WirelessDelay

Theresultsindicatethatthedelayresponseon either receivingor forwardingpacketsbythewirelessnodesusing VPNandwithoutVPNashavingalmostthe sameimpact,the averagedifferenceis about $40 \%$ betweenthesampleddelay indicatesthatat thefourthhouroftherunsimulationgivesa delayof $3.28 \mathrm{msecwithVPNand} 2.34 \mathrm{msec}$ ithoutVPN withadifferenceof0.94msec.

The load indicates the total traffic received and utilized across the wireless nodes. The graph shows the average variationof the WirelessloadwithandwithoutVPNacross thesenodes.Fig.9showsthesevariations.

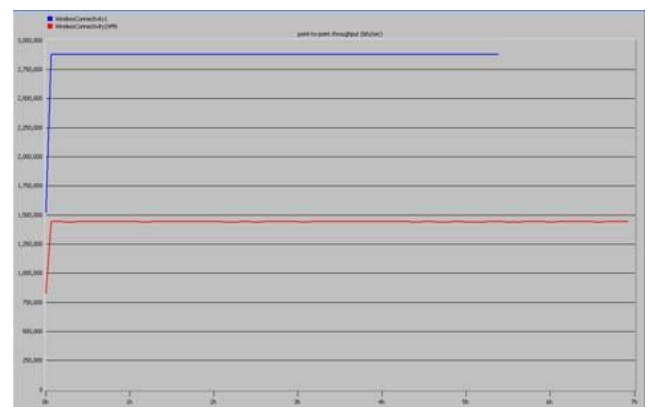

Fig.9WirelessLoadvariations 


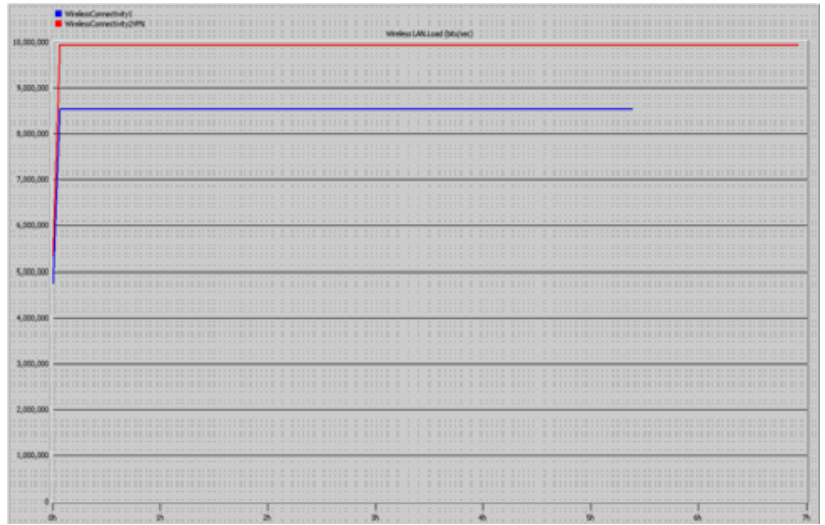

Theobtainedgraphindicatesan

averagedifferencebetween thetwosimulatedscenariosofabout $16 \%$,givenadifference of $174 \mathrm{~KB}$ atthe third minute of the run time simulation. Basedonthesampled VPNloadof9,953Kbits/Sec, and dataretrieved, atthethirdminuteof simulationrunindicatesa therestofthesimulationtime. gives8,558Kbits/Sec,anditremainsstablefor

Another analysis of VPN impact is the object which presents any node in the simulated scenario, as an object Access Point (1)has been analyzed toshow theimpact of VPNovera WirelessObjector nodes,especiallyattheMedia AccessDelay,whichindicatestheTotaltime(in seconds)that the packet is transmitted. The graph shown in Fig. 10 indicatestheresultsobtained.

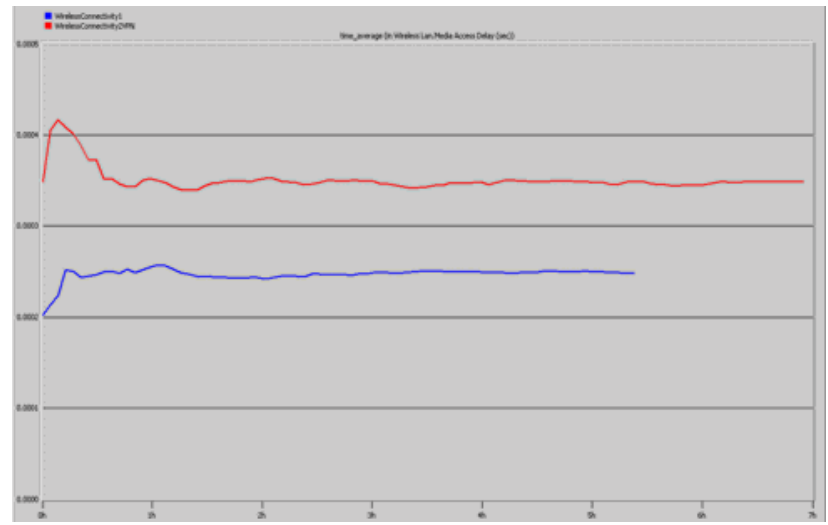

Fig.10WirelessnodesMediaAccessDelay

Thegraphshowsthat,theobjectdelaydifferencereached anaverageofabout $39 \%$, witha differenceof0.097msec. At thirdhourandfiftyminutesofthesimulatedrunwithVPN givesadelayof0.34msecandwithoutVPN,itgivesadelay of:0.25msec, which indicates averylowdelaydifference overtheAPobjectwithVPN.

Thelinkthroughputsarethethirdareatobeanalyzedafter the globaland object, which indicates the receiving and sendingofdatapackets. Theutilizedpointtopointlinkat the simulatedscenariosbetweentheAP1andRouterA (Referto Fig.11), whichshowstheresultsoftheutilizedlink. 
Fig.11InboundThroughput

Theresultsshowthatthe inboundthroughput, utilizationof VPNoverthe linkpresentsan overallaveragedifferenceof about50\%, atthe3rdminutethroughputoverthelinkwith VPN of 2,889Kbits/Secand Normal (No VPN) of

1,449Kbits/s. Theresultsshowahighthroughput

atthe

normal

scenariowithVPNbecauseofthedelaytimewithVPNand highdatadrop.

Using the same link, Fig. 12 shows the results of the outboundof throughputwithandwithoutVPN, whichdepicts the differenceandimpactof VPNwhenintroducedoverthe selectedpointtopointlink.

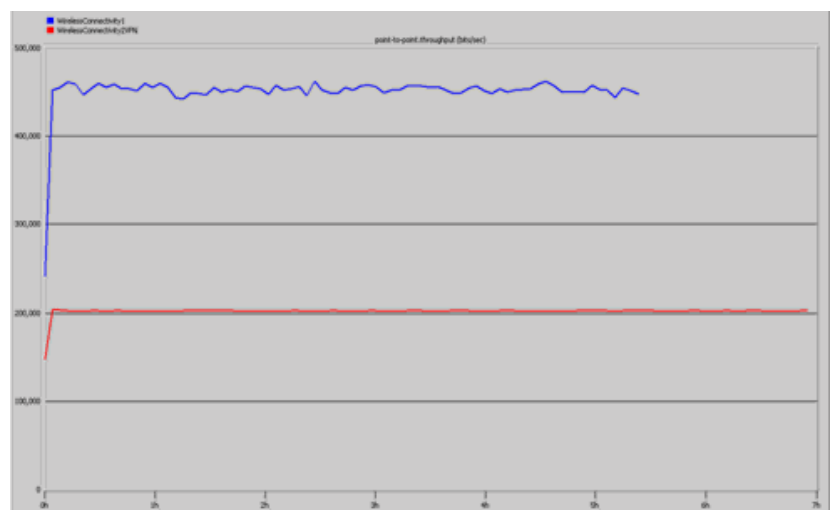

Fig.12OutboundThroughput

Theresultsclearlyshowthatthe outboundthroughput, utilizationofVPNoverthelinkpresentsan averagedifference ofabout $55 \%$.TheimpactofVPNshowsavaluelowerthan thatofthenormal WLAN scenario. Thisisbecause ofthe high delay time with VPN and High data Drop, which is almostsimilartotheinboundcase.

TableI,showsa summaryoftheobtainedresultscollected fromthe outcomesof thetwoscenarios. Theresultsshowa comparisontoindicatetheimpactof

Time,Load)ofVirtualPrivateNetworkoverwirelessLAN.

The performance impact has been analyzed from two differentparameters, the responsetimeandthe trafficload differencesandtheresultsaresummarizedbelow.

With regardto Response Time, given aDatabase server responsetimedifferenceof 2.37 seconds, whichisalmost $50 \%$ ofthenormalresponsetime, andtheresponsetimedifference

Of thewirelessnodes, whichreachesabout0.9milliseconds, givenanaverageof 40\%differenceofresponsetimedelay.Concluding an expected overall response time delay differences betweenthetwoscenarios, duetotheadditional VPN Processes, which been caused by the encryption processesandtheextraheadersaddedoneverypacket.

TABLEI

SUMMARY OFTHEOBTAINED RESULTSFORTHETWOSCENARIOS EXPLAINED IN TEXT

Time

(HR) Normal VPN Diff.\%

\section{GlobalResults}

\section{Conclusion}

Inthispaper, simulationof wirelessLANforIEEE802.11g protocol hasbeen done, andanalyzes impact ofintegrating Virtual Private Network technology to secure the flow of trafficbetweentheclientandthe serverfarmusingOPNET WLANutilityhas beencarriedout. TwoWirelessLAN scenarios have been considered and the results compared. These are Normal Extension toawired network and VPN overExtensiontoa wirednetwork. Theresultscollectedfrom thetwoscenarios,indicatethe impactof performance,mainly Response Time and Load, ofVirtual Private Network over wireless LAN. Roaming andhandover, which are closedDatabaseServer- ResponseTime(Sec) Wireless -Data 
Dropped (bits)

$\begin{array}{rrrrr}3: 08 & 4.98 & 7.35 & 2.37 & 47.5 \% \\ 3: 08 & 800 & 57710 & 56910 & 97 \%\end{array}$

Related,werenotconsideredinthecurrentwork.Inorderto

Implementroaming, the station should be continually scanning for the best signal of the available access points.Wireless - Delay $(\mathrm{Sec})$

-VPN

Wireless-Load(bits)

$-\mathrm{VPN}$

ObjectResults

(AccessPoint1)

MediaAccessDelay

3:08 $2.34 \times 10^{-3} \quad 3.25 \times 1$

$0^{-3}$

3:08 $\quad 8537180 \quad 993239$

0

3:08 $2.49 \times 10^{-4} \quad 3.46 \times 1$

$-4$

$0.91 \times 10^{-3} \quad 39 \%$

$1395210 \quad 16 \%$

$9.7 \times 10^{-5} \quad 39 \%$

Moreover, handoverrequirestheknowledge ofhandshaking

Process.However,in orderto realizean actualperformance impactofWLAN,it isimportanttoconsiderroamingand handoveraspectsinafuturework

\section{References}

$(\mathrm{Sec})$

LinkResults(AP1- RouterA) ThroughputInbound

(Bits) ${ }^{0}$

3:08 $2879920 \quad 144017$

$1439760 \quad 50 \%$

[1]

TheCentreforInternetsecurity,NetworkingBenchMark, ThesisTitle:

Wireless

NetworkingBenchmark,version1.0,April2005.

[2] P. K. Neelakantham, Villanova University, Thesis Title: Wireless

Networking Study of IEEE 802.11 Specification Communication Throughputoutbound

3:08 $448660 \quad 202010 \quad 246650 \quad 55 \%$

Networks,Summer2002.

(bits) 
[3] SelfStudyReportonPersonalAreaNetwork,Submittedby:H.Srikanth, Guid.

[4] National Institute Standards and Technology (NIST), Thesis Title:

Withregardto TrafficLoad, thetrafficloadoverthe WirelessLANwhichhasbeenproducedbytheVPN,shows that there isahigh traffic load difference indicated bythe collectedresults. Thetrafficloadon thewirelessnodesgivesa differenceof 174KBytestrafficandthe linkinboundgives atraffic of 180 bytes, andtheoutbound of 31 bytes. This indicatesahightrafficloadwhichhasahighimpactonthe datadropoverthe wirelessnodes. The datadropindicatesan overflow traffic over wireless nodes which need to be resolvedby usingtheIEEE802.11nprotocolinsteadof IEEE802.11g,whichhasahighbandwidthrateandincreases theEthernetbandwidthtoa Gigabit.ThisresultofVPN generating ahightrafficloadoverthewirelessLANnodes, whichimpactstheflowofdataoverthewirelessnodes.This is becauseoftheencryptionprocessesandthe added authenticationheadersforeachpacketsent.

TableIIgivesa bandwidthrequirementofvarious applications[13], whichwillhelporganizationsdecidehow muchbandwidthrequiredto use for VPNovertheirWireless LANbasedontheaboveoutcomes.

TABLEII

BANDWIDTH REQUIREMENTSFORVARIOUSAPPLICATIONS

\begin{tabular}{ll} 
Application & Rate \\
\hline E-mail & 2.4to9.6Kbits/Sec \\
Database & Upto1 MBits/Sec
\end{tabular}

Wireless Network Security 802.11, Bluetooth and Handheld Devices, By:TomKarygiannisandLesOwens,2002.

[5] DISAFieldSecurityOperations,FinalDraftWirelessSecurityTechnical ImplementationGuide,Version4,Release0.3,18thAugust2005.

N.Edde,SecurityComplete,SecondEdition, 2002.

[7] Stockholm's University, Master Thesis: Security, Centrefor an Enterprise,ByHuxiodong,Feb2005.

[8] Schlumberger Information Solutions, Houston, WhitePaperof:Virtual Network Solutions forRemote Access, Comparison between SSLand IPSec,2004.

[9] R.Myers,TechnologyIndustry,inCommunication News,ArticleTitle: CombineVPNandEncryption-WirelessSecurity,2003.

[10] MercurionSystemInc, InformationTechnologyConsultingandSupport Services, Whitepaper:UsingVPNto SecureWLANTraffic,2004, http://www.mercurionsystems.com.

[11] B.LewisandP.T. Davis,ElectronicBook,WileyPublishing,Inc, 2004. [12] NS Simulator for beginners, University De Los Andes, Sophia-

Antipolis, 2003.

[13] OPNETModelerAcceleratingNetworkR\&D,Opnet11.0product documentation,www.opnet.com/support.

[14] Cisco System Inc, Cisco Wireless Security, Chapter:8, www.searchnetworking.techtarget.com/search networking/downloads /chapter08.pdf.

[15] G. Bianchi, L. Fratta, M. Oliveri, "Performance Evaluation and Enhancement of the CSMA/CA MAC Protocol for 802.11 Wireless LANs",Proc.PIMRC1996,October1996,Taipei,Taiwan,pp.392-396.

[16] G. Bianchi, "Performance Analysis of the IEEE 802.11 Distributed

Coordination Function," IEEE Journal on Selected Areas in

Communications, Vol.18,No.3,pp.535-547,Mar.2000.

[17] N.T.DaoandR.A.Malaney,"ANewMarkovModelforNon-Saturated

802.11 Networks", 5th IEEE Consumer Communications and

NetworkingConference, 2008 .

DocumentImaging

CompressedVideo

10to100MBits/Sec

2to10MBits/Sec 